\title{
Article \\ Some Explicit Expressions for Twisted Catalan-Daehee Numbers
}

\author{
Dongkyu Lim (D)
}

Department of Mathematics Education, Andong National University, Andong 36729, Korea; dklim@anu.ac.kr

\begin{abstract}
In this paper, the author considers the twisted Catalan numbers and the twisted CatalanDaehee numbers, which are arisen from $p$-adic fermionic integrals and $p$-adic invariant integrals, respectively. We give some explicit identities and properties for those twisted numbers and polynomials by using $p$-adic integrals or generating functions.
\end{abstract}

Keywords: twisted Catalan numbers; twisted Catalan-Daehee numbers; $p$-adic fermionic integrals; $p$-adic invariant integrals

MSC: Primary 05A19; 11B83

Citation: Lim, D. Some Explicit

Expressions for Twisted

Catalan-Daehee Numbers. Symmetry

2022, 14, 189. https://doi.org/

10.3390/sym14020189

Academic Editors: Junesang Choi

and Djurdje Cvijović

Received: 20 November 2021

Accepted: 14 January 2022

Published: 19 January 2022

Publisher's Note: MDPI stays neutral with regard to jurisdictional claims in published maps and institutional affiliations.

Copyright: (C) 2022 by the author. Licensee MDPI, Basel, Switzerland. This article is an open access article distributed under the terms and conditions of the Creative Commons Attribution (CC BY) license (https:// creativecommons.org/licenses/by/ $4.0 /)$.

\section{Introduction}

The Catalan numbers were first introduced by the Mongolian mathematician Ming Antu in 1730, even though they were named after the French-Belgian mathematician Eugène Charles Catalan (1814-1894). The Catalan numbers $C_{n}$ are given [1-4] by the generating function

$$
\sum_{n=0}^{\infty} C_{n} t^{n}=\sum_{n=0}^{\infty} \frac{1}{n+1}\left(\begin{array}{c}
2 n \\
n
\end{array}\right) t^{n}
$$

We note that

$$
\begin{aligned}
\sqrt{1-4 t} & =1-2 \sum_{m=0}^{\infty}\left(\begin{array}{c}
2 m \\
m
\end{array}\right) \frac{1}{m+1} t^{m+1} \\
& =1-2 \sum_{m=0}^{\infty} C_{m} t^{m+1}
\end{aligned}
$$

They satisfy the recurrence relations

$$
C_{0}=1, \quad C_{n}=\sum_{m=0}^{n-1} C_{m} C_{n-1-m,} \quad(n \geq 1) .
$$

The Catalan numbers form a sequence of positive integers

$$
1,1,2,5,14,42,132,429,1430,4862,16,796,58,786,208,012, \cdots
$$

which is asymptotic to $4^{n} / n^{\frac{3}{2}} \sqrt{\pi}$, as $n$ tends to $\infty$, and appears in various counting problems. For example, $C_{n}$ is the number of Dyck words of length $2 n$, the number of balanced $n$ pairs of parentheses, the number of mountain ranges you can form with $n$ upstrokes and downstrokes that all stay above the original line, the number of diagonal-avoiding paths of length $2 n$ from the upper left corner to the lower right corner in a grid of $n \times n$ squares, and the number of ways in which $n+1$ factors can be completely parenthesized. It is also the number of ways an $(n+2)$-gon can be cut into $n$ triangles, the number of permutations of $\{1,2, \cdots, n\}$ that avoid the pattern 123 , the number of ways to tile a stair step shape of height $n$ with $n$ rectangles, etc. (see $[3,4]$ ). For the rest of this section, we recall the necessary facts that are needed throughout the paper.

Let $p$ be a fixed odd prime number. Throughout this paper, $\mathbb{Z}_{p}, \mathbb{Q}_{p}$ and $\mathbb{C}_{p}$ will denote the ring of $p$-adic integers, the field of $p$-adic rational numbers and the completion 
of the algebraic closure of $\mathbb{Q}_{p}$. The $p$-adic norm is normalized as $|p|_{p}=\frac{1}{p}$. Let $f(x)$ be a continuous $\mathbb{C}_{p}$-valued function on $\mathbb{Z}_{p}$. Then the fermionic $p$-adic integral on $\mathbb{Z}_{p}$ is defined $[1,2,5-7]$ by

$$
\begin{aligned}
I_{-1}(f) & =\int_{\mathbb{Z}_{p}} f(x) d \mu_{-1}(x) \\
& =\lim _{N \rightarrow \infty} \sum_{x=0}^{p^{N}-1} f(x)(-1)^{x} .
\end{aligned}
$$

From (4), we note that

$$
I_{-1}\left(f_{1}\right)=-I_{-1}(f)+2 f(0), \text { where } f_{1}(x)=f(x+1) .
$$

Remarkably, in [2], Dolgy et al. represented Catalan numbers by the fermionic $p$-adic integral on $\mathbb{Z}_{p}$ as follows:

$$
\begin{aligned}
\int_{\mathbb{Z}_{p}}(1-4 t)^{\frac{x}{2}} d \mu_{-1}(x) & =\frac{2}{1+\sqrt{1-4 t}} \\
& =\sum_{n=0}^{\infty} \frac{1}{n+1}\left(\begin{array}{c}
2 n \\
n
\end{array}\right) t^{n}=\sum_{n=0}^{\infty} C_{n} t^{n} .
\end{aligned}
$$

where $t \in \mathbb{C}_{p}$ with $|t|_{p}<p^{-\frac{1}{p-1}}$.

On the other hand, for any uniformly differentiable function $f: \mathbb{Z}_{p} \rightarrow \mathbb{C}_{p}$, the $p$-adic invariant integral $I_{1}(f)$ is given $[7,8]$ by

$$
I_{1}(f)=\int_{\mathbb{Z}_{p}} f(x) d \mu(x)=\lim _{N \rightarrow \infty} \frac{1}{p^{N}} \sum_{x=0}^{p^{N}-1} f(x) .
$$

From (6), we can derive the following integral equation:

$$
I_{1}\left(f_{1}\right)=I_{1}(f)+f^{\prime}(0), \text { where } f^{\prime}(0)=\left.\frac{d f(x)}{d x}\right|_{x=0} .
$$

Motivated by the relation in (7), Kim-Kim [6] applied $f(x)=(1-4 t)^{\frac{x}{2}}$ on the $p$-adic invariant integral on $\mathbb{Z}_{p}$,

$$
\int_{\mathbb{Z}_{p}}(1-4 t)^{\frac{x}{2}} d \mu(x)=\frac{\frac{1}{2} \log (1-4 t)}{\sqrt{1-4 t}-1}
$$

for $|t|_{p}<p^{-\frac{1}{p-1}}$. Using (8), the authors defined the Catalan-Daehee numbers by the generating function

$$
\sum_{n=0}^{\infty} d_{n} t^{n}=\frac{\frac{1}{2} \log (1-4 t)}{\sqrt{1-4 t}-1}
$$

By (1) and (9), Dolgy et al. [1] connected the Catalan numbers and the Catalan-Daehee numbers as follows:

$$
d_{n}= \begin{cases}1, & \text { if } n=0 \\ \frac{4^{n}}{n+1}-\sum_{m=0}^{n-1} \frac{4^{n-m-1}}{n-m} C_{m}, & \text { if } n \geq 1\end{cases}
$$

For $n \in \mathbb{N}$, let $T_{p}$ be the $p$-adic locally constant space defined by

$$
T_{p}=\bigcup_{n \geq 1} C_{p^{n}}=\lim _{n \rightarrow \infty} C_{p^{n}}
$$


where $C_{p^{n}}=\left\{w \mid w^{p^{n}}=1\right\}$ is the cyclic group of order $p^{n}$

Catalan-Daehee numbers and polynomials were introduced in [6] and considered the family of linear differential equations arising from the generating function of those numbers in order to derive some explicit identities involving Catalan-Daehee numbers and Catalan numbers. In [7], several properties and identities associated with Catalan-Daehee numbers and polynomials were derived by utilizing umbral calculus techniques. Dolgy et al. gave some new identities for those numbers and polynomials derived from $p$-adic Volkenborn integrals on $\mathbb{Z}_{p}$ in [1]. Later, Kim et al. studied Catalan numbers and Catalan-Daehee numbers in various ways, (see $[1,2,6,7,9,10])$.

The purpose of this paper is to construct a new type of numbers and polynomials, the twisted Catalan numbers and polynomials and the twisted Catalan-Daehee numbers and polynomials, and to investigate some properties and identities of these polynomials. We note that the twisted numbers are considered by Kim, as an analogue of Bernoulli numbers and their applications in [11]. Later, several twisted numbers and polynomials are treated in the literatures (see [12-14]).

\section{Twisted Catalan Numbers}

In this section, we assume that $t \in \mathbb{C}_{p}$ with $|t|_{p}<p^{-\frac{1}{p-1}}$. For $w \in T_{p}$, let us take $f(x)=w^{x}(1-4 t)^{\frac{x}{2}}$ in (5). Then, we have

$$
\begin{aligned}
\int_{\mathbb{Z}_{p}} w^{x}(1-4 t)^{\frac{x}{2}} d \mu_{-1}(x) & =\frac{2}{w \sqrt{1-4 t}+1} \\
& =\frac{2}{1-w^{2}+4 w^{2} t}(1-w \sqrt{1-4 t}) .
\end{aligned}
$$

Motivated from (10), we consider the twisted Catalan numbers which are given by the generating function to be

$$
\frac{2}{1-w^{2}+4 w^{2} t}(1-w \sqrt{1-4 t})=\sum_{n=0}^{\infty} C_{n, w} t^{n} .
$$

By (10) and (11), specially $w=1$, we can recover the Catalan numbers in (1),

$$
\frac{1}{2 t}(1-\sqrt{1-4 t})=\sum_{n=0}^{\infty} C_{n} t^{n}
$$

From (10) or (11), we derive the following:

$$
\begin{aligned}
\frac{2}{1-w^{2}+4 w^{2} t} & (1-w \sqrt{1-4 t})=\frac{2}{1-w^{2}+4 w^{2} t}\left(1+w \sum_{l=0}^{\infty}\left(\begin{array}{c}
2 l \\
l
\end{array}\right) \frac{1}{2 l-1} t^{l}\right) \\
& =\frac{2}{1-w^{2}}\left(\frac{1}{1+\frac{4 w^{2}}{1-w^{2}}}\right)\left(1+w \sum_{l=0}^{\infty}\left(\begin{array}{c}
2 l \\
l
\end{array}\right) \frac{1}{2 l-1} t^{l}\right) \\
& =\frac{2}{1-w^{2}}\left(\sum_{m=0}^{\infty}\left(\frac{4 w^{2}}{1-w^{2}}\right)^{m}(-1)^{m} t^{m}\right)\left(1+w \sum_{l=0}^{\infty}\left(\begin{array}{c}
2 l \\
l
\end{array}\right) \frac{1}{2 l-1} t^{l}\right) \\
& =\frac{2}{1-w^{2}} \sum_{m=0}^{\infty}\left(\frac{4 w^{2}}{1-w^{2}}\right)^{m}(-1)^{m} t^{m}+\frac{2 w}{1-w^{2}} \sum_{n=0}^{\infty} \sum_{l=0}^{n}\left(\begin{array}{c}
2 l \\
l
\end{array}\right) \frac{(-1)^{n-l}}{2 l-1}\left(\frac{4 w^{2}}{1-w^{2}}\right)^{n-l} t^{n} \\
& =\sum_{n=0}^{\infty}\left[\frac{2}{1-w^{2}}\left\{\left(-\frac{4 w^{2}}{1-w^{2}}\right)^{n}+w \sum_{l=0}^{n}\left(\begin{array}{c}
2 l \\
l
\end{array}\right) \frac{(-1)^{n-l}}{2 l-1}\left(\frac{4 w^{2}}{1-w^{2}}\right)^{n-l}\right\} t^{n} .\right.
\end{aligned}
$$

Therefore, by (11) and (12), we obtain the following. 
Theorem 1. For $w \in T_{p}$ and $n \geq 0$, we have

$$
\begin{aligned}
C_{n, w} & =\frac{2}{1-w^{2}}\left\{\left(-\frac{4 w^{2}}{1-w^{2}}\right)^{n}+w \sum_{l=0}^{\infty}\left(\begin{array}{c}
2 l \\
l
\end{array}\right) \frac{(-1)^{n-l}}{2 l-1}\left(\frac{4 w^{2}}{1-w^{2}}\right)^{n-l}\right\} \\
& =\frac{2 w}{1-w^{2}} \sum_{l=1}^{n}\left(\begin{array}{c}
2 l \\
l
\end{array}\right) \frac{(-1)^{n-l}}{2 l-1}\left(\frac{4 w^{2}}{1-w^{2}}\right)^{n-l}+\frac{2}{1-w^{2}}\left(-\frac{4 w^{2}}{1-w^{2}}\right)^{n} .
\end{aligned}
$$

For example,

$$
\begin{aligned}
C_{0, w} & =\frac{2}{1+w^{\prime}} \\
C_{1, w} & =\frac{2}{1-w^{2}}\left(w \frac{4 w^{2}}{1-w^{2}}-\frac{4 w^{2}}{1-w^{2}}+2 w\right) \\
& =\frac{2}{1-w^{2}} \frac{2 w-2 w^{2}}{1+w}=\frac{4 w}{(1+w)^{2}}, \\
C_{2, w} & =\frac{2}{1-w^{2}}\left(-w\left(\frac{4 w^{2}}{1-w^{2}}\right)^{2}-2 w \frac{4 w^{2}}{1-w^{2}}+\frac{w}{3} 6+\left(\frac{4 w^{2}}{1-w^{2}}\right)^{2}\right) \\
& =\frac{2}{1-w^{2}} \frac{(1-w)\left(6 w^{2}+2 w\right)}{\left(1+w^{2}\right.}=\frac{12 w^{2}+4 w}{(1+w)^{3}}, \\
C_{3, w} & =\frac{2}{1-w^{2}}\left(\left(-\frac{4 w^{2}}{1-w^{2}}\right)^{3}+w\left(\frac{4 w^{2}}{1-w^{2}}\right)^{3}+2 w\left(\frac{4 w^{2}}{1-w^{2}}\right)^{3}+4 w\right) \\
& =\frac{2}{1-w^{2}} \frac{4(1-w)\left(5 w^{3}+4 w^{2}+w\right)}{(1+w)^{3}}=\frac{8\left(5 w^{3}+4 w^{2}+w\right)}{(1+w)^{4}}, \\
C_{4, w} & =\frac{70 w^{4}+94 w^{3}+50 w^{2}+10 w}{(1+w)^{5}},
\end{aligned}
$$

Note that

$$
\begin{aligned}
\lim _{w \rightarrow 1} C_{0, w} & =1=C_{0}, \quad \lim _{w \rightarrow 1} C_{1, w}=1=C_{1}, \quad \lim _{w \rightarrow 1} C_{2, w}=2=C_{2}, \\
\lim _{w \rightarrow 1} C_{3, w} & =5=C_{3}, \quad \lim _{w \rightarrow 1} C_{4, w}=14=C_{4}, \\
& \ldots \\
\lim _{w \rightarrow 1} C_{n, w} & =\lim _{w \rightarrow 1} \frac{2}{1-w}\left\{\left(-\frac{4 w^{2}}{1-w^{2}}\right)^{n}+w \sum_{l=0}^{\infty}\left(\begin{array}{c}
2 l \\
l
\end{array}\right) \frac{(-1)^{n-l}}{2 l-1}\left(\frac{4 w^{2}}{1-w^{2}}\right)^{n-l}\right\} \\
& =\left(\begin{array}{c}
2 n \\
n
\end{array}\right) \frac{1}{n+1}=C_{n}, \quad(n \geq 0) .
\end{aligned}
$$

\section{Twisted Catalan-Daehee Numbers}

Motivated by Dolgy et al. in [1], we consider the twisted Catalan-Daehee numbers by the $p$-aidc invariant integral on $\mathbb{Z}_{p}$ for the function $f(x)=w^{x}(1-4 t)^{\frac{x}{2}}$,

$$
\int_{\mathbb{Z}_{p}} w^{x}(1-4 t)^{\frac{x}{2}} d \mu(x)=\frac{\frac{1}{2} \log (1-4 t)}{w \sqrt{1-4 t}-1}=\sum_{n=0}^{\infty} d_{n, w} t^{n}
$$

where $t \in \mathbb{C}_{p}$ with $|t|_{p}<p^{-\frac{1}{p-1}}$ and $w \in T_{p}$. 
Remark 1. When we apply the function $f(x)=w^{x}(1-4 t)^{\frac{x}{2}}$ to the $p$-adic invariant integral on $\mathbb{Z}_{p}$, then by (6), we have

$$
\int_{\mathbb{Z}_{p}} w^{x}(1-4 t)^{\frac{x}{2}} d \mu(x)=\frac{\log w+\frac{1}{2} \log (1-4 t)}{w \sqrt{1-4 t}-1} .
$$

Since $w \in T_{p}$, we have $w^{p^{n}}=1$ for some $n \in \mathbb{N}$, which implies that $\log w=\frac{1}{p^{n}} \log w^{p^{n}}=$ $\frac{1}{p^{n}} \log 1=0$. So we defined the generating function for the twisted Catalan-Daehee numbers as (14).

From (9) and (11), we derive the following

$$
\begin{aligned}
\frac{\frac{1}{2} \log (1-4 t)}{w \sqrt{1-4 t}-1} & =\frac{1}{2} \log (1-4 t) \frac{w \sqrt{1-4 t}+1}{w^{2}(1-4 t)-1} \\
& =\frac{1}{4} \log (1-4 t) \frac{2(1-w \sqrt{1-4 t}-2)}{1-w^{2}+4 w^{2} t} \\
& =\frac{1}{4} \log (1-4 t) \frac{2(1-w \sqrt{1-4 t})}{1-w^{2}+4 w^{2} t}-\log (1-4 t) \frac{1}{1-w^{2}+4 w^{2} t} \\
& =\frac{1}{4} \log (1-4 t) \sum_{m=0}^{\infty} C_{m, w} t^{m}-\frac{\log (1-4 t)}{1-w^{2}} \frac{1}{1+\frac{4 w^{2}}{1-w^{2}}} \\
& =-\frac{1}{4}\left(\sum_{l=0}^{\infty} \frac{4^{l+1} t^{l+1}}{l+1}\right)\left(\sum_{m=0}^{\infty} C_{m, w} t^{m}\right)+\frac{1}{1-w^{2}} \sum_{l=0}^{\infty} \frac{4^{l+1}}{l+1} t^{l+1} \sum_{m=0}^{\infty}\left(\frac{-4 w^{2}}{1-w^{2}}\right)^{m} t^{m} \\
& =-\frac{1}{4} \sum_{n=1}^{\infty}\left(\sum_{m=0}^{n-1} \frac{4^{n-m}}{n-m} C_{m, w}\right)^{n}+\frac{1}{1-w^{2}} \sum_{n=1}^{\infty} \sum_{m=0}^{n-1} \frac{4^{n-m}}{n-m}(-1)^{m}\left(\frac{4 w^{2}}{1-w^{2}}\right)^{m} t^{n} .
\end{aligned}
$$

Therefore, by (11) and (15), we obtain a relation between the twisted Catalan-Daehee numbers and the twisted Catalan numbers.

Theorem 2. For $w \in T_{p} \backslash\{1\}$ and $n \geq 0$, we have

$$
d_{n, w}=\sum_{m=0}^{n-1}\left(\frac{-4^{n-m-1}}{n-m} C_{m, w}+\frac{(-1)^{m}}{1-w^{2}} \frac{4^{n-m}}{n-m}\left(\frac{4 w^{2}}{1-w^{2}}\right)^{m}\right)
$$

For example, we have

$$
\begin{aligned}
d_{0, w} & =0, \\
d_{1, w} & =-\frac{2}{w-1}, \quad d_{2, w}=-\frac{4 w-1}{(w-1)^{2}}, \\
d_{3, w} & =-\frac{46 w^{2}-50 w+16}{(w-1)^{3}}, \quad d_{4, w}=-\frac{44 w^{3}-76 w^{2}+50 w-12}{(w-1)^{4}}, \\
& \ldots .
\end{aligned}
$$
on $\mathbb{Z}_{p}$.

We define the twisted Catalan-Daehee polynomials by the $p$-adic invariant integral

$$
\begin{aligned}
\int_{\mathbb{Z}_{p}} w^{y}(1-4 t)^{\frac{x+y}{2}} d \mu(y) & =\frac{\frac{1}{2} \log (1-4 t)}{w \sqrt{1-4 t}-1}(1-4 t)^{\frac{x}{2}} \\
& =\sum_{n=0}^{\infty} d_{n, w}(x) t^{n}
\end{aligned}
$$


Now, we want to give relations between the twisted Catalan-Daehee polynomials and the twisted Bernoulli polynomials. The twisted Bernoulli polynomials are defined by the generating function

$$
\frac{t}{w e^{t}-1} e^{t x}=\sum_{n=0}^{\infty} B_{n, w}(x) \frac{t^{n}}{n !} .
$$

When $x=0, B_{n, w}=B_{n, w}(0)$ are the twisted Bernoulli numbers, which are defined and studied by Kim in [11].

For $w \in T_{p}$ and $|t|_{p}<p^{-\frac{1}{p-1}}$, we have

$$
\begin{aligned}
\sum_{n=0}^{\infty} d_{n, w}(x) t^{n} & =\int_{\mathbb{Z}_{p}} w^{y}(1-4 t)^{\frac{x+y}{2}} d \mu(y) \\
& =\sum_{m=0}^{\infty} \frac{1}{2^{m}} m !(\log (1-4 t))^{m} \int_{\mathbb{Z}_{p}} w^{y}(x+y)^{m} d \mu(y) \\
& =\sum_{m=0}^{\infty} 2^{-m} B_{m, w}(x) \sum_{n=m}^{\infty} S_{1}(n, m) \frac{(-4 t)^{n}}{n !} \\
& =\sum_{n=0}^{\infty}\left(\sum_{m=0}^{n} 2^{-m} B_{m, w}(x) S_{1}(n, m) \frac{(-4)^{n}}{n !}\right) t^{n}
\end{aligned}
$$

Therefore, by (17), we obtain the following.

Theorem 3. For $w \in T_{p}$ and $n \geq 0$, we have

$$
d_{n, w}(x)=\frac{(-1)^{n}}{n !} \sum_{m=0}^{n} 2^{2 n-m} B_{m, w}(x) S_{1}(n, m),
$$

where $S_{1}(n, m)$ is the Stirling numbers of the first kind.

Replacing $t$ by $\frac{1}{4}\left(1-e^{2 t}\right)$ in (16), we have

$$
\begin{aligned}
\sum_{m=0}^{\infty} B_{m, w}(x) \frac{t^{m}}{m !} & =\sum_{n=0}^{\infty} \frac{(-1)^{n} d_{n, w}(x)}{4^{n}}\left(e^{2 t}-1\right)^{n} \\
& =\sum_{n=0}^{\infty} \frac{(-1)^{n} d_{n, w}(x)}{4^{n}} \sum_{m=n}^{\infty} S_{2}(m, n) \frac{2^{m} t^{m}}{m !} \\
& =\sum_{m=0}^{n}\left(\sum_{n=0}^{m}(-1)^{n} 2^{m-2 n} n ! S_{2}(m, n) d_{n, w}(x)\right) \frac{t^{m}}{m !} .
\end{aligned}
$$

From (18), we obtain the inversion formula of the Theorem 3.

Theorem 4. For $w \in T_{p}$ and $n \geq 0$, we have

$$
B_{n, w}(x)=\sum_{m=0}^{n}(-1)^{m} 2^{n-2 m} m ! S_{2}(n, m) d_{m, w}(x),
$$

where $S_{2}(n, m)$ is the Stirling numbers of the second kind. 
Alternatively, $d_{n, w}(x)$ can be expressed as follows:

$$
\begin{aligned}
\sum_{n=0}^{\infty} d_{n, w}(x) t^{n} & =\int_{\mathbb{Z}_{p}} w^{y}(1-4 t)^{\frac{x+y}{2}} d \mu(y) \\
& =\sum_{n=0}^{\infty}(-4)^{n} \int_{\mathbb{Z}_{p}} w^{y}\left(\frac{x+y}{2}\right)_{n} d \mu(y) \frac{t^{n}}{n !} \\
& =\sum_{n=0}^{\infty}(-4)^{n} \int_{\mathbb{Z}_{p}} w^{y}\left(\begin{array}{c}
\frac{x+y}{2} \\
n
\end{array}\right) d \mu(y) t^{n} .
\end{aligned}
$$

By (17) and (19), we have the following

Corollary 1. For $w \in T_{p}$ and $n \geq 0$, we have

$$
\begin{aligned}
\int_{\mathbb{Z}_{p}} w^{y}\left(\begin{array}{c}
\frac{x+y}{2} \\
n
\end{array}\right) d \mu(y) & =(-1)^{n} \frac{d_{n, w}(x)}{4^{n}} \\
& =\frac{1}{n !} \sum_{m=0}^{\infty} 2^{-m} B_{m, w}(x) S_{1}(n, m) .
\end{aligned}
$$

For $\lambda \in \mathbb{Z}_{p},|t|_{p}<p^{-\frac{1}{p-1}}$ and $w \in T_{p}$, the twisted $\lambda$-Daehee polynomials $D_{n, w}(x \mid \lambda)$ are defined by the generating function

$$
\frac{\lambda \log (1+t)}{w(1+t)^{\lambda}-1}(1+t)^{x}=\sum_{n=0}^{\infty} D_{n, w}(x \mid \lambda) \frac{t^{n}}{n !} .
$$

Thus, by (7), we obtain

$$
\begin{aligned}
\int_{\mathbb{Z}_{p}} w^{y}(1+t)^{\lambda y+x} d \mu(y) & =\frac{\lambda \log (1+t)}{w(1+t)^{\lambda}-1}(1+t)^{x} \\
& =\sum_{n=0}^{\infty} D_{n, w}(x \mid \lambda) \frac{t^{n}}{n !} .
\end{aligned}
$$

In particular, the twisted $\lambda$-Daehee numbers $D_{n, w}(\lambda)=D_{n, w}(0 \mid \lambda)$ are given by

$$
\begin{aligned}
\frac{\lambda \log (1+t)}{w(1+t)^{\lambda}-1} & =\sum_{n=0}^{\infty} D_{n, w}(\lambda) \frac{t^{n}}{n !} \\
& =\int_{\mathbb{Z}_{p}} w^{y}(1+t)^{\lambda y} d \mu(y) .
\end{aligned}
$$

We note that $D_{0, w}(\lambda)=0$. In particularly, $w=1, D_{n, \lambda}=D_{n, 1}(\lambda)$ is the $n$th $\lambda$-Daehee numbers in [1].

From (14) and (20), we have

$$
\begin{aligned}
\sum_{n=0}^{\infty} d_{n, w} t^{n} & =\frac{\frac{1}{2} \log (1-4 t)}{w \sqrt{1-4 t}-1} \\
& =\sum_{n=0}^{\infty} D_{n, w}\left(\frac{1}{2}\right)(-4)^{n} \frac{t^{n}}{n !} .
\end{aligned}
$$

By (22), we have

$$
n ! d_{n, w}=(-4)^{n} D_{n, w}\left(\frac{1}{2}\right), \quad(n \geq 0) .
$$


From (1) and (16), we have

$$
\begin{aligned}
\sum_{n=0}^{\infty} d_{n, w} t^{n} & =\frac{\frac{1}{2} \log (1-4 t)}{w^{2}(1-4 t)-1}(w \sqrt{1-4 t}+1) \\
& =\left(\frac{\log (1-4 t)}{w^{2}(1-4 t)-1}\right)\left(\frac{1+w}{2}-w \sum_{m=0}^{\infty} C_{m} t^{m+1}\right) \\
& =\frac{\frac{1+w}{2} \log (1-4 t)}{w^{2}(1-4 t)-1}-\frac{w \log (1-4 t)}{w^{2}(1-4 t)-1} \sum_{m=0}^{\infty} C_{m} t^{m+1} \\
& =\sum_{n=0}^{\infty}(-4)^{n} D_{n, w^{2}}\left(\frac{1+w}{2}\right) \frac{t^{n}}{n !}-\left(\sum_{l=0}^{\infty}(-4)^{l} D_{l, w^{2}}(w) \frac{t^{l}}{l !}\right) \sum_{m=0}^{\infty} C_{m} t^{m+1} \\
& =\sum_{n=0}^{\infty}(-4)^{n} D_{n, w^{2}}\left(\frac{1+w}{2}\right) \frac{t^{n}}{n !}-\sum_{n=1}^{\infty}\left(\sum_{m=0}^{n-1}(-4)^{n-m-1} \frac{D_{n-m-1, w^{2}}(w)}{(n-m-1) !} C_{m}\right) t^{n} \\
& =\sum_{n=1}^{\infty}\left(\frac{(-4)^{n}}{n !} D_{n, w^{2}}\left(\frac{1+w}{2}\right)-\sum_{m=0}^{n-1}(-4)^{n-m-1} \frac{D_{n-m-1, w^{2}}(w)}{(n-m-1) !} C_{m}\right) t^{n} .
\end{aligned}
$$

Theorem 5. For $w \in T_{p}$ and $n \geq 1$, we have

$$
d_{n, w}=\frac{(-4)^{n}}{n !} D_{n, w^{2}}\left(\frac{1+w}{2}\right)-\sum_{m=0}^{n-1}(-4)^{n-m-1} \frac{D_{n-m-1, w^{2}}(w)}{n !(n-m-1) !} C_{m} .
$$

Alternatively, (14) can be represented.

$$
\begin{aligned}
\sum_{n=0}^{\infty} d_{n, w} t^{n} & =\frac{\frac{1}{2} \log (1-4 t)}{w^{2}(1-4 t)-1}(w \sqrt{1-4 t}+1) \\
& =\frac{1+w}{2} \sum_{n=0}^{\infty}(-4)^{n} D_{n, w^{2}}(1) \frac{t^{n}}{n !}-w\left(\sum_{l=0}^{\infty}(-4)^{l} D_{l, w^{2}}(1) \frac{t^{l}}{l !}\right)\left(\sum_{m=0}^{\infty} C_{m} t^{m+1}\right) \\
& =\frac{1+w}{2} \sum_{n=0}^{\infty}(-4)^{n} D_{n, w^{2}}(1) \frac{t^{n}}{n !}-w \sum_{n=1}^{\infty}\left(\sum_{m=0}^{n-1} \frac{(-4)^{n-m-1}}{(n-m-1) !} D_{n-m-1, w^{2}}(1) C_{m}\right) t^{n} \\
& =\sum_{n=1}^{\infty}\left(\frac{1+w}{2} \frac{(-4)^{n}}{n !} D_{n, w^{2}}(1)-w \sum_{m=0}^{n-1} \frac{(-4)^{n-m-1}}{(n-m-1) !} D_{n-m-1, w^{2}}(1) C_{m}\right) t^{n} .
\end{aligned}
$$

Therefore, by (23), we obtain the following.

Theorem 6. For $w \in T_{p}$ and $n \geq 1$, we have

$$
d_{n, w}=\frac{(-4)^{n}}{n !} \frac{1+w}{2} D_{n, w^{2}}(1)-w \sum_{m=0}^{n-1} \frac{(-4)^{n-m-1}}{(n-m-1) !} D_{n-m-1, w^{2}}(1) C_{m}
$$

Using (16) and (21), we observe the following

$$
\begin{aligned}
\sum_{n=0}^{\infty} d_{n, w}(x) t^{n} & =\int_{\mathbb{Z}_{p}} w^{y}(1-4 t)^{\frac{x+y}{2}} d \mu(y) \\
& =\sum_{n=0}^{\infty} D_{n, w}\left(\frac{x}{2} \mid \frac{1}{2}\right)(-4)^{n} \frac{t^{n}}{n !} .
\end{aligned}
$$

From (24), we have

$$
n ! d_{n, w}(x)=(-4)^{n} D_{n, w}\left(\frac{x}{2} \mid \frac{1}{2}\right), \quad(n \geq 0)
$$


and it follows that

$$
\begin{aligned}
\sum_{n=0}^{\infty} d_{n, w}(x) t^{n} & =\int_{\mathbb{Z}_{p}} w^{y}(1-4 t)^{\frac{x+y}{2}} d \mu(y) \\
& =\sum_{n=0}^{\infty} \int_{\mathbb{Z}_{p}}(-4)^{n} w^{y}\left(\begin{array}{c}
\frac{x+y}{2} \\
n
\end{array}\right) d \mu(y) t^{n}
\end{aligned}
$$

By (26), we have

$$
d_{n, w}(x)=(-4)^{n} \int_{\mathbb{Z}_{p}} w^{y}\left(\begin{array}{c}
\frac{x+y}{2} \\
n
\end{array}\right) d \mu(y)
$$

We note that

$$
\begin{aligned}
\sum_{n=0}^{\infty} d_{n, w}(x) t^{n} & =\frac{\frac{1}{2} \log (1-4 t)}{w \sqrt{1-4 t}-1}(1-4 t)^{\frac{x}{2}} \\
& =\left(\sum_{l=0}^{\infty} d_{l, w} t^{l}\right)\left(\sum_{m=0}^{\infty}\left(\begin{array}{c}
\frac{x}{2} \\
m
\end{array}\right)(-4)^{m} t^{m}\right) \\
& =\sum_{n=0}^{\infty}\left(\sum_{m=0}^{n}\left(\begin{array}{c}
\frac{x}{2} \\
m
\end{array}\right)(-4)^{m} d_{n-m, w}\right) t^{n}
\end{aligned}
$$

From (28), we obtain another expression for the twisted Catalan-Daehee polynomials.

$$
d_{n, w}(x)=\sum_{m=0}^{n}\left(\begin{array}{c}
\frac{x}{2} \\
m
\end{array}\right)(-4)^{m} d_{n-m, w}
$$

Therefore, by (25), (27) and (29), we have the following.

Theorem 7. For $w \in T_{p}$ and $n \geq 0$, we have

$$
\begin{aligned}
d_{n, w}(x) & =\sum_{m=0}^{n}\left(\begin{array}{c}
\frac{x}{2} \\
m
\end{array}\right)(-4)^{m} d_{n-m, w} \\
& =\frac{(-4)^{n}}{n !} D_{n, w}\left(\frac{x}{2} \mid \frac{1}{2}\right) \\
& =\int_{\mathbb{Z}_{p}}(-4)^{n} w^{y}\left(\begin{array}{c}
\frac{x+y}{2} \\
n
\end{array}\right) d \mu(y) .
\end{aligned}
$$

For the case of twisted Catalan-Daehee polynomials, we can connect to the twisted $\lambda$-Daehee polynomials as follows;

$$
\begin{aligned}
\sum_{n=0}^{\infty} d_{n, w}(x) t^{n} & =\frac{\frac{1}{2} \log (1-4 t)}{w^{2}(1-4 t)-1}(w \sqrt{1-4 t}+1)(1-4 t)^{\frac{x}{2}} \\
& =\frac{\frac{1}{2} \log (1-4 t)}{w^{2}(1-4 t)-1}(1-4 t)^{\frac{x+1}{2}}+\frac{\frac{1}{2} \log (1-4 t)}{w^{2}(1-4 t)-1}(1-4 t)^{\frac{x}{2}} \\
& =\sum_{n=0}^{\infty}(-4)^{n} D_{n, w^{2}}\left(x+1 \mid \frac{w}{2}\right) \frac{t^{n}}{n !}+\sum_{n=0}^{\infty}(-4)^{n} D_{n, w^{2}}\left(x \mid \frac{1}{2}\right) \frac{t^{n}}{n !} \\
& =\sum_{n=0}^{\infty}\left((-4)^{n} D_{n, w^{2}}\left(x+1 \mid \frac{w}{2}\right)+(-4)^{n} D_{n, w^{2}}\left(x \mid \frac{1}{2}\right)\right) \frac{t^{n}}{n !} .
\end{aligned}
$$

Thus by (30), we have the following.

Theorem 8. For $w \in T_{p}$ and $n \geq 0$, we have

$$
d_{n, w}(x)=\frac{(-4)^{n}}{n !}\left(D_{n, w^{2}}\left(x+1 \mid \frac{w}{2}\right)+D_{n, w^{2}}\left(x \mid \frac{1}{2}\right)\right)
$$




\section{Conclusions}

To summarize, by means of $p$-adic integrals on $\mathbb{Z}_{p}$, we introduced a new type of numbers and polynomials, the twisted Catalan numbers and polynomials and the twisted CatalanDaehee numbers and polynomials, and obtained several explicit expressions and identities related to them. In Section 2, the twisted Catalan numbers were introduced with the help of a fermionic $p$-adic integral on $\mathbb{Z}_{p}$. We derived explicit expressions of the twisted Catalan numbers, as a rational function in $w$ in Theorem 1 . We also obtained a relation between the twisted Catalan-Daehee numbers and the twisted Catalan numbers on Theorem 2. In Section 3, we introduced the twisted Catalan-Daehee numbers and polynomials and obtained several explicit expressions and identities related to them. In more detail, we expressed the twisted Catalan-Daehee polynomials in terms of the twisted Bernoulli polynomials and Stirling numbers of the first kind in Theorem 3. We also derived an identity involving the twisted Bernoulli numbers, twisted Catalan-Daehee numbers and Stirling numbers of the second kind in Theorem 4. After that, we obtained the explicit expressions for the twisted Catalan-Daehee polynomials, which involve the twisted $\lambda$-Daehee numbers and Catalan numbers in Theorems 5 and 6. In addition, in Theorems 7 and 8, we showed the relationships between the twisted Catalan-Daehee polynomials and the twisted $\lambda$-Daehee polynomials.

To conclude, there are various methods for studying special polynomials and numbers, including: generating functions, symmetric identities, computational algorithms, combinatorial methods, umbral calculus, differential equations, probability theory, integral representations, including the Riemann integral, contour integral and $p$-adic integrals, and analytic number theory, (see [1-20]). For the further study on the twisted Catalan numbers and the twisted Catalan-Daehee numbers, we could extend our works to use differential equations, degenerate version or higher order concepts on those numbers and polynomials. In [20], Kucukoglu et al. constructed generating functions for new classes of Catalantype numbers and polynomials. Using these functions and their functional equations, they gave various new identities and relations involving these numbers and polynomials, and other classes of special numbers, polynomials, and functions. Some infinite series representations, including the Catalan-type numbers and combinatorial numbers, were investigated. Moreover, some recurrence relations and computational algorithms for these numbers and polynomials were provided. By implementing these algorithms in the Python programming language, they illustrated the Catalan-type numbers and polynomials with their plots under the special conditions. They also gave some derivative formulas for these polynomials. Applying the Riemann integral, contour integral, Volkenborn integral, and fermionic $p$-adic integral to these polynomials. It is one of future projects to continue to study the twisted Catalan numbers and the twisted Catalan-Daehee numbers along the line of the direction of Catalan-type paper.

Funding: The work of D. Lim was partially supported by the National Research Foundation of Korea (NRF) grant funded by the Korean government (MSIT) NRF-2021R1C1C1010902.

Institutional Review Board Statement: Not applicable.

Informed Consent Statement: Not applicable.

Data Availability Statement: Not applicable.

Acknowledgments: The author would like to thank the referees for their comments and suggestions which improved the original manuscript in its present form.

Conflicts of Interest: The author declares that he has no conflict of competing interests.

\section{References}

1. Dolgy, D.V.; Jang, G.-W.; Kim, D.S.; Kim, T. Explicit expressions for Catalan-Daehee numbers. Proc. Jangjeon Math. Soc. 2017, 20, 1-9.

2. Kim, T. A note on Catalan numbers associated with $p$-adic integral on $\mathbb{Z}_{p}$. Proc. Jangjeon Math. Soc. 2016, 19, 493-501.

3. Koshy, T. Catalan Numbers with Applications; Oxford University Press: Oxford, UK, 2009.

4. Stanley, R.P. Catalan Numbers; Cambridge University Press: New York, NY, USA, 2015. 
5. Kim, T. q-Volkenborn integration. Russ. J. Math. Phys. 2002, 9, 288-299.

6. Kim, T.; Kim, D.S. Differential equations associated with Catalan-Daehee numbers and their applications. Revista de la Real Academia de Ciencias Exactas Físicas y Naturales. Serie A Matemáticas. 2017, 111, 1071-1081. [CrossRef]

7. Kim, T.; Kim, D.S.; Seo, J.-J. Symmetric identities for an analogue of Catalan polynomials. Proc. Jangjeon Math. Soc. 2016, 19, 515-521.

8. Kim, D.S.; Kim, T. Daehee numbers and polynomials. Appl. Math. Sci. (Ruse) 2013, 7, 5969-5976. [CrossRef]

9. Kim, D.S.; Kim, T. A new approach to Catalan numbers using differential equations. Russ. J. Math. Phys. 2017, 24, 465-475. [CrossRef]

10. Kim, T.; Kim, D.S. Some identities of Catalan-Daehee polynomials arising from umbral calculus. Appl. Comput. Math. 2017, 16, 177-189.

11. Kim, T. An analogue of Bernoulli numbers and their applications. Rep. Fac. Sci. Engrg. Saga Univ. Math. 1994, $22,21-26$.

12. Jang, L.-C. A family of Barnes-type multiple twisted $q$-Euler numbers and polynomials related to Fermionic $p$-adic invariant integrals on $\mathbb{Z}_{p}$. J. Comput. Anal. Appl. 2011, 13, 376-387.

13. Moon, E.-J.; Rim, S.-H.; Jin, J.-H.; Lee, S.-J. On the symmetric properties of higher-order twisted $q$-Euler numbers and polynomials. Adv. Differ. Equ. 2010, 2010, 765259. [CrossRef]

14. Park, J.-W. On the $\lambda$-Daehee polynomials with q-parameter. J. Comput. Anal. Appl. 2016, 20, 11-20.

15. Cagman, A. Explicit solutions of powers of three as sums of three Pell numbers based on Baker's type inequalities. Turkish J. Ineq. 2021, 5, 93-103.

16. Cagman, A. Repdigits as Product of Fibonacci and Pell numbers. Turkish J. Sci. 2021, 6, 31-35.

17. Srivastava, H.M.; Choi, J. Zeta and q-Zeta Functions and Associated Series and Integrals; Elsevier Science Publisher B.V.: Amsterdam, The Netherlands, 2012.

18. Kim, D.S.; Kim, T. Triple symmetric identities for $w$-Catalan polynomials. J. Korean Math. Soc. 2017, 54, $1243-1264$.

19. Kucukoglu, I.; Simsek, B.; Simsek, Y. An approach to negative hypergeometric distribution by generating function for special numbers and polynomials. Turk. J. Math. 2019, 43, 2337-2353. [CrossRef]

20. Kucukoglu, I.; Simsek, B.; Simsek, Y. New classes of Catalan-type numbers and polynomials with their applications related to p-adic integrals and computational algorithms. Turk. J. Math. 2020, 44, 2337-2355. [CrossRef] 